\title{
A high performance scanning force microscope head design
}

\author{
Steven M. Clark \\ Division of Biology, California Institute of Technology, Pasadena, California 91125 \\ John D. Baldeschwieler \\ A. A. Noyes Laboratory of Chemical Physics, Division of Chemistry, California Institute of Technology, \\ Pasadena, California 91125
}

(Received 2 November 1992; accepted for publication 19 November 1992)

\begin{abstract}
A stable and highly sensitive scanning force microscope head design is presented. The head provides an implementation of the optical lever detection method in which mechanical vibration noise has been minimized.
\end{abstract}

\section{INTRODUCTION}

Since its inception in 1985, the atomic force microscope ${ }^{l}$ [also known as the scanning force microscope (SFM)] has held great promise for imaging nonconducting samples with subnanometer resolution. Early attempts to image relatively inelastic samples such as graphite, crystals of ionic salts, and semiconductors yielded atomic resolution data. These data provided reason to believe that SFM might be applied easily to the study of biomaterials. However, as more compliant samples were examined, it became clear that several factors limit resolution on elastic samples. Among these factors are (i) unavoidable forces involved in tip-sample interaction, (ii) tip shape, (iii) sample motion, both scan induced and thermal, and (iv) instrument and detection noise. Progress has been made in all of these areas; for example, imaging with both sample and cantilever immersed in liquid to reduce meniscus forces, ${ }^{2}$ electron beam deposition of tips to increase tip sharpness, ${ }^{3}$ imaging with specially prepared substrates to increase sample adhesion during imaging, ${ }^{4}$ as well as efforts to image at low temperature to eliminate Brownian motion of the sample. ${ }^{5}$ Other investigators have focused on sources of instrument noise and their reduction. ${ }^{6}$ This article describes a design to reduce mechanical noise and increase the stability of a SFM stage so that it exceeds the requirements for imaging biomaterials.

Establishing the force level acceptable for biomolecular imaging is difficult; it depends on the sample, preparation procedures, imaging environment, and other factors. Theoretical discussions of tolerable forces for biological samples advocate applied forces in the $10^{-10}-10^{-11} \mathrm{~N}$ range., We have used the more conservative $10^{-11} \mathrm{~N}$ figure as a design goal for the SFM stage presented. One consequence of this force criterion is that, for stiff micromachined cantilevers (which have spring constants $\approx 1 \mathrm{~N} / \mathrm{m}$ ) motions larger than $0.1 \AA$ are unacceptable. We have designed a SFM head that achieves stability and vibrational amplitudes less than this level. The body of this article describes the design of the SFM head and discusses the most important factors considered during the design process.

\section{DESIGN OVERVIEW}

A diagram of the SFM head is shown in Fig. 1. Most commercial instruments are designed such that the sample is attached to the scanning element and moved while the probe remains stationary (except for small vertical deflections). In contrast, with our design the cantilever is scanned in a raster fashion over the stationary sample. This configuration was chosen for two reasons; first, it allows the head to be used in a "stand alone" mode in which it is used without the base, allowing the investigation of samples which are too large to be mounted on the SFM base. Second, in the case of samples on transparent substrates, it allows optical accessibility from the other side of the sample. This type of architecture allows the head to be used on an inverted epifluorescence type of microscope by using the microscope stage as the SFM base, thus making possible direct correlation between sample features in fluorescence microscopy and SFM.

\section{MECHANICAL DESIGN}

The ideas at the foundation of the mechanical design are few and simple. They are (i) minimize the number of parts, (ii) keep structures small and mechanically stiff, and (iii) make sure that vibrations induced by seismic and acoustic disturbances are common mode to the structure. There was no effort made to use dissipative materials in the design because the philosophy was to eliminate excitation sources through acoustic shielding and vibration isolation as required, rather than design a system with low $Q$ resonances in which vibrations might still enter the region of experimental interest.

The SFM head consists of only one major part, the head itself, which serves as a stiff platform to rigidly couple the input telescope, the piezo tube, and the detector. This makes most motions induced by acoustic and seismic stimuli common mode to the structure and minimizes their effect.

To achieve our second design goal we used parts of small physical dimensions and mounted them in a rigid fashion. The only partial exception is the photodetector support which takes the form of a post with a 1/2-in.-diam bore for the return beam path from the cantilever. Although this structure is large, it is integral to the head and quite stiff. The frequencies of its lowest resonant modes are well above the regions of experimental interest. Despite attempts to excite transverse vibrations of the photodiode support, we have been unable to observe its motion. This 


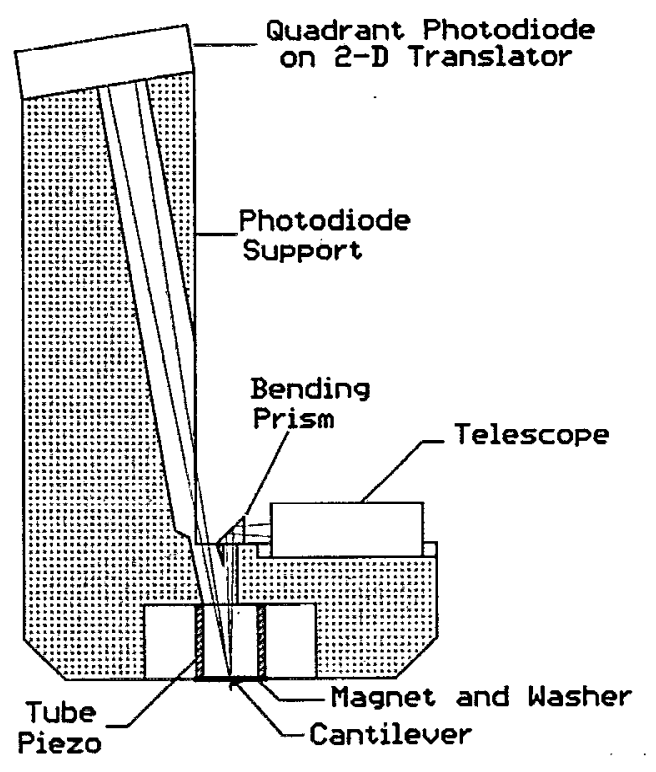

FIG. 1. A diagram of the scanning force microscope head. This simplified diagram of the SFM head indicates the relative positions of important components. For clarity, the base and the head support (approach) screws have been omitted.

leads one to believe that resonant motion of the support is, in fact, negligible.

The piezo tube is mounted in such a way as to avoid compliant coupling to the head. The electrodes are etched back from the edges of the tube to create a small area (a strip 0.005-in.-wide on both ends of the piezo) of insulating ceramic material. This prevents electrical shorting and allows the piczo to be glued directly to both the head and the magnet which holds the cantilever assembly, thus avoiding the use of layers of insulating materials which may be elastic.

The cantilever support consists of a small steel wedge soldered to a steel washer. The cantilever die is positioned near the wedge such that the beam reflected from the back of the cantilever is centered in the bore of the photodiode support. During the optical alignment process the die is held and positioned by means of a vacuum chuck with a multiaxis adjustment fixture. Once the proper cantilever position has been found, the die is glued to the wedge to fix its position. While this procedure is somewhat cumbersome, it allows for the compensation of cantilever warpage or other changes in the position of the return beam (such as those from a liquid cantilever environment). In practice we find that the alignment time taken for this instrument is about the same as that required for mounting and alignment tips in the commercial instruments we have used. The elimination of optical adjustments in the beam paths is the major compromise in ease of use which has been made in order to eliminate potential sources of mechanical noise.

\section{OPTICAL DESIGN}

Although the scanned cantilever architecture has attractive features, it places severe constraints on the input optics. These constraints are (i) the beam must be focused

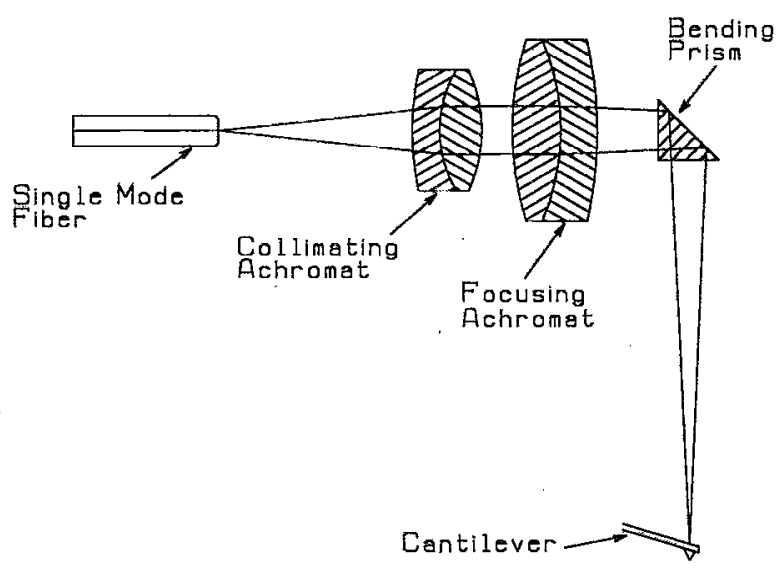

FIG. 2. A diagram of the single-mode optical fiber input optics. The telescope is composed of two achromats. It uses a connectorized singlemode optical fiber as input and focuses the light to $\mathrm{a} \approx 10-\mu \mathrm{m}$ diameter spot at $\approx 1.2 \mathrm{~cm}$ from the output lens. This provides a spot of the required dimensions and, because the telescope has no local heat source, the position of the spot is extremely stable.

to a small enough spot that the entire beam is incident on the back of the cantilever, even during large lateral motions; (ii) the focused spot must have a depth of focus large enough to accommodate the full range of cantilever motion in the $Z$ axis; and (iii) the pointing stability of the input beam must be excellent to avoid introducing noise into the SFM cxperiment. This last constraint was found to be particularly important in previous SFM head designs constructed in our laboratory. In some cases instrument performance was compromised by thermal drift of components in the input beam path. In order to demonstrate the flexibility of this head design, we have used two different light input schemes.

The object of the first scheme, shown in Fig. 2, is to remove heat sources from the head. To accomplish this we removed the laser from the head assembly by using a single-mode optical fiber to couple the laser output to the input optics. In addition to avoiding thermal transients, as might be induced by an integral laser diode, this scheme also provides good vibration isolation and allows the use of different lasers.

The use of a single mode fiber (SMF) for the input beam dictates that a telescope be provided to focus the beam onto the cantilever. The SMF has a $4-\mu \mathrm{m}$ diam core and a numerical aperture of $\approx 0.1$. The desired beam diameter incident on the back of the cantilever is $\approx 10 \mu \mathrm{m}$, the depth of the focus must be $>2 \mu \mathrm{m}$ and the focus must lie $\approx 1.5 \mathrm{~cm}$ from the front surface of the second lens. These figures impose a maximum lateral magnification of two and demand diffraction-limited performance of the telescope. This was achieved through the use of two achromats; the first collimates the output of the fiber and optimally matches the collimated beam diameter to the second lens, and the second serves to focus the beam on the cantilever. Ray traces of the telescope predict the $1 / e^{2}$ spot diameter at the cantilever position $(1.2 \mathrm{~cm}$ from the last surface) to be $10 \mu \mathrm{m}$ and the depth of focus to be $\approx 25 \mu \mathrm{m}$. These figures agree with the results of a Gaussian optical 


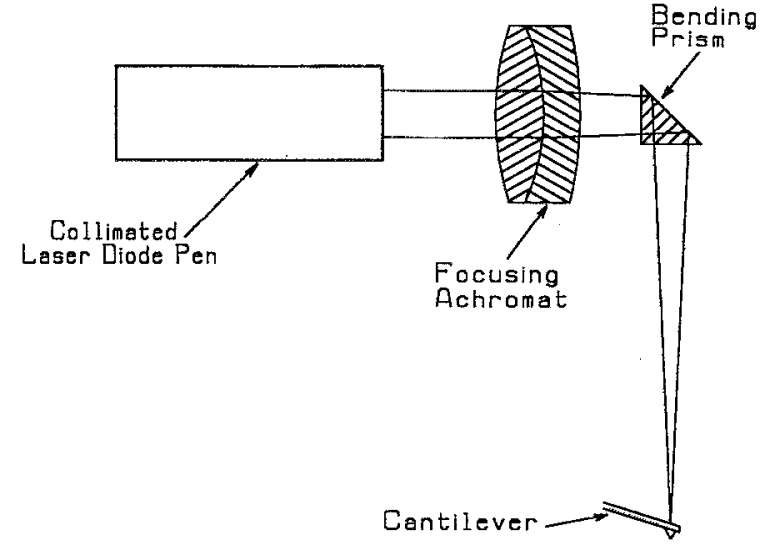

FIG. 3. A diagram of the laser diode input optics. Light from a visible laser diode pen is focused onto the back of the cantilever by an achromat. Only one lens is required because the output of the laser diode pen is collimated. The focused spot at the cantilever is elliptical with a major axis of $\approx 20 \mu \mathrm{m}$ and a minor axis of $\approx 10 \mu \mathrm{m}$. In our implementation of this scheme the laser diode pen mounts directly in the lens housing and this assembly is substituted for the telescope assembly.

analysis of the system which give the spot diameter as 10 $\mu \mathrm{m}$ and a depth of field of $\approx 30 \mu \mathrm{m}$.

The telescope rests in a "vee" groove machined into the head that serves to constrain its motions except axial rotation and translation. After optimizing the spot diameter on the cantilever by axial translation of the telescope, a small spring clamp holds it in position. This "semikinematic" mounting provides very stable and repeatable positioning of the telescope even during focus adjustment. The only other optical component in the input beam path is the bending prism which deflects the beam, by total internal reflection, so that it is coaxial with the piezoelectric ceramic lube. (Because the acceptance angle of the prism exceeds the beam convergence angle, the criterion for total internal reflection is met by all rays.) A prism was chosen for this application instead of a mirror because it does not require a mount if glued directly to the head and so provides an inherent stability in defining the optical path. The input optics perform satisfactorily as judged by the apparent beam diameter and stability as viewed on the cantilever at $40 \times$ magnification and by the Gaussian spot reflected from the cantilever.

The second light input configuration uses an integral laser diode. This scheme, shown in Fig. 3, is conceptually the same as that first used for SFM by Meyer and Amer, ${ }^{9}$ and is often found in commercial instruments. In our implementation a collimated laser diode "bullet" is used as a light source and the output of the "bullet" is focused onto the cantilever by an achromat. From ray traces of this system we estimate the focused spot to be elliptical with a major axis of $\approx 20 \mu \mathrm{m}$ and a minor axis of $\approx 10 \mu \mathrm{m}$.

As with any optical lever based SFM, the beam reflected from the back of the cantilever provides a sensitive measure of cantilever deflection. In this design, a quadrant photodiode is used to sense the position of the reflected beam and provides both torsional and vertical deflection signals. The fine positioning of the photodiode can be ad- justed by means of a two-dimensional short travel translator mechanism. There are no optical elements between the cantilever and the photodiode. Thus the only source of beam motion is the motion of the cantilever (disregarding pointing noise of the input optics which is negligible). The optical lever gain has been made as large as possible to provide maximal sensitivity and increases the signal-tonoise ratio. The reflected beam from the cantilever is divergent, because it is focused on the cantilever, and it is this divergence which places a practical limit on the path

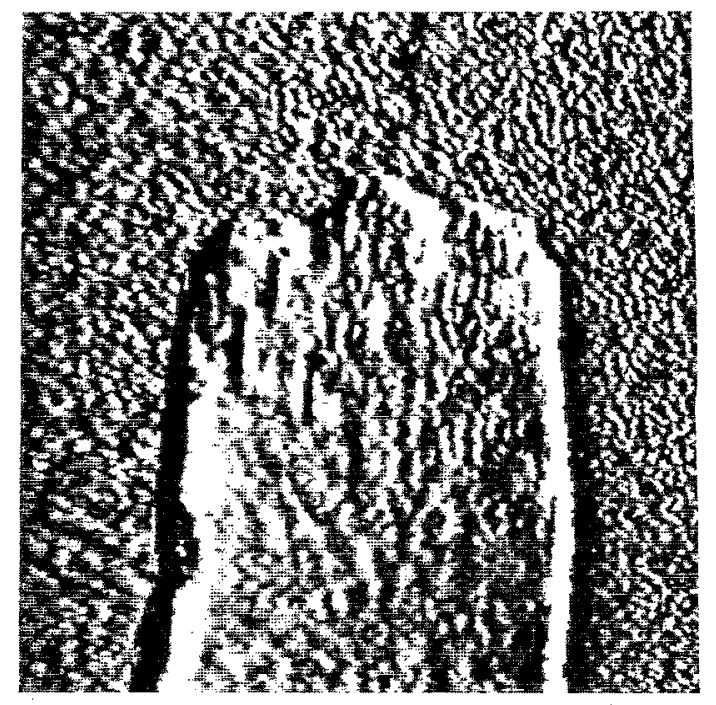

a)

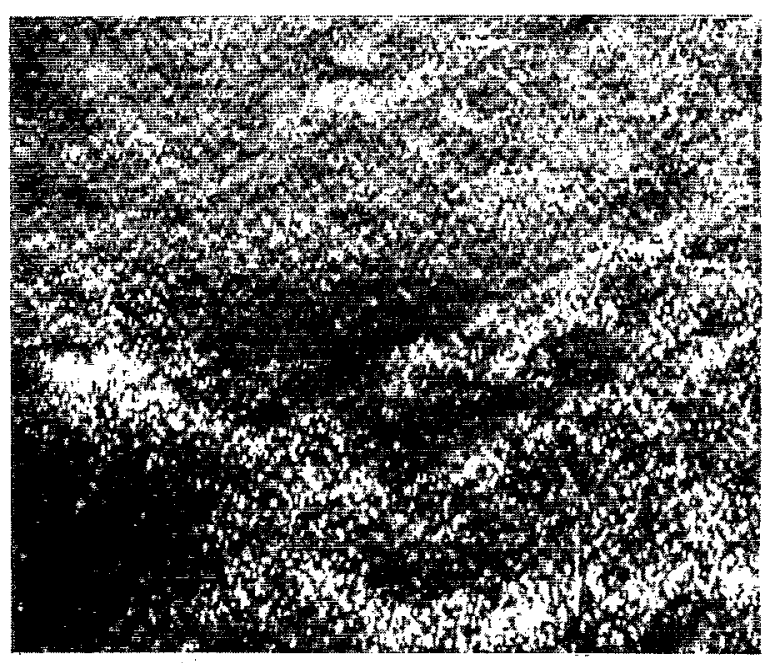

b)

FIG. 4. Images of microcrystals of beef liver catalase. These images were taken in air with an electron beam deposited "super" tip. Both images were acquired using forces of $\approx 0.1 \mathrm{nN}$ and have a sampled resolution of $250 \times 250$ pixels. They are displayed with brightness proportional to both topographical height and slope after tilt was removed by plane subtraction. (a) A low magnification image (1.5 $\mu \mathrm{m} \times 1.5 \mu \mathrm{m}$ scan range) of a single microcrystal of beef liver catalase on a glass substrate. The crystal has a plate morphology with obvious defects in its surface. (b) A higher magnification image ( $340 \mathrm{~nm} \times 300 \mathrm{~nm}$ range) of a region of the same catalasc microcrystal. In this image the $\approx 10 \mathrm{~nm}$ period of the crystal is visible, however the resolution is inadequate to visualize the tetrameric structure of the individual catalase molecules which comprise the crystal. 
length from the cantilever to the photodiode. To allow the path to be as long as possible, a large photodiode is used to collect all of the return beam. The photodiode we use has $\mathrm{a} \approx 12-\mathrm{mm}$ aperture allowing a path length of $\approx 12 \mathrm{~cm}$ from the back of the cantilever to the face of the photodiode. In addition to enabling large optical lever gain, the increased size of the beam at the detector alleviates any concerns about local saturation of the photodiode active surface. Because the bandwidth we require is less than $\approx 100 \mathrm{kHz}$, the photodiode is used in the photovoltaic mode to minimize noise.

\section{PERFORMANCE OF THE HEAD}

In order to demonstrate the capability of the head design, in Fig. 4 we show images of a microcrystal of bovine catalase. Imaging of protein microcrystals requires that only small forces be applicd to the sample; thus thcy provide a good test of the performance of the SFM head design. The first image, Fig. 4(a), shows a low magnification scan $(1.5 \mu \mathrm{m} \times 1.5 \mu \mathrm{m})$ taken in air of a single protein microcrystal. The plate morphology of the crystal is similar to that of other larger crystals found in the sample by light and electron microscopy (data not shown). In several places large defects in the crystal (e.g., concavities in the surface) can be seen. In Fig. 4(b), a higher magnification image is shown in which the crystalline repeat can be seen. The characteristic dimension of the repeat is $\approx 10 \mathrm{~nm}$, which agrees well with literature values. ${ }^{10-12} \mathrm{Although}$ catalase has a tetrameric structure, this image does not exhibit adequate resolution for these features to be seen. The im- ages of Fig. 4 were taken in air at a sampled resolution of $250 \times 250$ pixels using a commercial cantilever with an electron beam deposited tip. The force applied to the sample, as calculated from cantilever deflection, cantilever geometry, and materials constants, was less than $1 \mathrm{nN}$ for both of the images shown. The authors will gladly provide more detailed documentation to interested readers.

\section{ACKNOWLEDGMENTS}

The authors wish to thank Charles F. Spence for stimulating discussions and Dr. Jean-Paul Revel and Michael G. Youngquist for a critical reading of the manuscript. Financial support for this work was provided by Abbott Laboratories, Inc., the National Institutes of Health (Training Grant GM 07617) and the United States Public Health Service (Training Grant HG00021).

${ }^{1}$ G. Binnig, Ch. Gerber, and C. F. Quate, Phys. Rev. Lett. 56, 930 (1986).

${ }^{2}$ H. G. Hansma et al., Science 2561180 (1992).

${ }^{3}$ Y. Akama, E. Nishimura, A. Sakai, and H. Murakami, J. Vac. Sci. Technol. A 8, 429 (1990).

${ }^{4} \mathrm{C}$. Bustamante et al., Biochemistry 31, 22 (1992).

${ }^{5}$ C. B. Prater et al., J. Vac. Sci. Technol. B 9, 989 (1991).

${ }^{6} \mathrm{~S}$. M. Clark et al., Rev. Sci. Instrum. (in press).

${ }^{7}$ B. N. J. Persson, Chem. Phys. Lett. 141, 366 (1987).

${ }^{8}$ K. E. Drexler, J. Vac. Sci. Technol. B 9, 1394 (1991).

${ }^{9}$ G. Meyer and N. M. Amer, Appl. Phys. Lett. 53, 1045 (1988).

${ }^{10}$ M. R. N. Murthy et al., J. Mol. Biol. 152, 465 (1981).

${ }^{11}$ I. Fita et al., Acta Cryst. B 42, 497 (1986).

${ }^{12}$ T. Furuno, K. Ulmer, and H. Sasabe, Microsc. Res. Tech. 21, 32 (1992). 